\title{
Reproduzierbarkeit dermatologischer Befunde aus epidemiologischen Studien
}

\author{
U. Krämer ${ }^{1,2}$, H. Behrendt ${ }^{2,3}$, R. Dolgner ${ }^{1}$, T. Schäfer ${ }^{2}$, A. Steup ${ }^{1}$, G. A. Wiesmüller ${ }^{1}$, J. Ring $^{2}$ \\ ${ }^{1}$ Medizin. Institut für Umwelthygiene, Düsseldorf; ${ }^{2}$ Klinik u. Poliklinik für Dermatologie u. Allergologie der TU München; \\ ${ }^{3}$ Abtlg. für experimentelle Dermatologie, Hautklinik am Universitätskrankenhaus Eppendorf, Hamburg
}

Zum Erfassen dermatologischer Befunde in epidemiologischen Studien wird in der Klinik für Dermatologie und Allergologie der TU München ein standardisierter Befundbogen eingesetzt. Auf diesem können neun Atopiestigmata (tiefreichender Haaransatz, Gesichtsblässe, periokuläre Schatten, Dennie-Morgan-Falte, Herthoge-Zeichen, Ichthyosis-Hand, Ichthyosis-Fuß, Sebostase und weißer Dermographismus) in den Ausprägungen ,nicht vorhanden“, „schwach“, „mäßig“ und „stark vorhanden“ eingetragen werden. Das Vorliegen der Minimalmanifestationen Cheilitis sicca, Mundwinkelrhagaden, Ohrläppchenrhagaden, Pulpite seche und Pityriasis alba wird ebenfalls dokumentiert.

In zwei der bisher durchgeführten epidemiologischen Studien wurde die- ser Bogen wenigstens zweimal bei demselben Probanden eingesetzt. 28 Erwachsene (12 Männer, 16 Frauen; mittleres Alter 40,0 Jahre) wurden im Oktober 1995 und im April 1996 jeweils von einem Arzt untersucht. 119 Kinder (60 Jungen, 59 Mädchen; mittleres Alter 8,3 Jahre) wurden im September 1993 und im März 1994 von teilweise unterschiedlichen Ärzten untersucht. Der Kappa-Koeffizient wurde zur Beurteilung der Übereinstimmung zwischen den Befundungen in den jeweils beiden Untersuchungsrunden gewählt. In der Kinderstudie zeigte sich, daß die Variabilität in der Zuordnung von Atopiestigmata und Minimalmanifestationen bei der Untersuchung durch einen Arzt oder durch mehrere Ärzte ähnlich war. Eine Dennie-Morgan-Falte wurde als einzige der erfaß- ten Größen auch in den unterschiedlichen Ausprägungen stabil erfaßt. Werden die Beurteilungskategorien auf „vorhanden“, „,nicht vorhanden“ reduziert, so erwies sich die Beurteilung des tiefreichenden Haaransatzes und der Ichthyosis-Hand ebenfalls noch als stabil. Bei den Erwachsenen war das Maß an Übereinstimmung in der Regel höher. Hier wurden zusätzlich noch die Gesichtsblässe und jeweils ein Score aus Atopiestigmata und aus Minimalmanifestationen stabil beurteilt. Möglicherweise sind Stigmata und Manifestationen bei Erwachsenen stabiler. In einer nachfolgenden Studie soll untersucht werden, inwiefern sich die Reproduzierbarkeit der Erfassung auch bei Kindern durch eine noch eingehendere Schulung der Ärzte verbessern läßt.

\section{Regressionsbäume zur Beurteilung von Interaktionen und Subpopulationen der Prädiktoren des kindlichen Sonnenbrands}

\author{
B. Lausen ${ }^{1}$, A. Pfahlberg ${ }^{2,3}$, O. Gefeller², K.F. Koelmel ${ }^{3}$ \\ ${ }^{1}$ Department of Medical Statistics and Evaluation, Imperial College, London (GB); \\ ${ }^{2}$ Abteilung Medizinische Statistik, Universität Göttingen; ${ }^{3}$ Universitäts-Hautklinik Göttingen
}

Die Methode der Klassifikations- und Regressionsbäume (Classification And Regression Trees $=$ CART) wird oft als ein Verfahren zur Selektion von Prädiktoren benutzt. Die rekursive Konstruktion der Regressionsbäume liefert ein Modell von möglichen Interaktionen zwischen den Prädiktoren bzw. eine explorative Suche nach auffälligen Subpopulationen.

Bei jeder Rekursion wird die Population anhand eines maximal selektierten Split-Kriteriums aufgeteilt. Wir verwenden hierbei als Split-Kriterium den adjustierten P-Wert der maximal selektierten 2-Stichproben-Wilcoxon-Rangstatistik. Dies erlaubt die Analyse von Prädikto- ren, die auf unterschiedlichen Skalen gemessen werden. Wir wenden diese Methode beispielhaft auf eine von der Universitäts-Hautklinik Göttingen durchgeführte Untersuchung zur Evaluation gesundheitsaufklärerischer und -erzieherischer Bemühungen, das Wissen und Verhalten von Eltern hinsichtlich Sonnenschutz bei ihren Kinder zu verbessern, an. Die mittels schriftlicher Befragung erhobenen Daten zweier zeitversetzter Querschnittsstudien an allen 56 Göttinger Kindergärten im Frühjahr 1992 und Herbst 1994 (Gesamtstichprobenumfang 2491 Fragebögen) werden insbesondere auf möglicher Interaktionen der Prädiktoren des kindlichen Son- nenbrands analysiert. Der Unterschied zwischen den Befragungen erweist sich als dominierender Prädiktor des kindlichen Sonnenbrands: Vor der Kampagne zeigen die betrachteten Regressionsbäume eine vielschichtige Struktur der Prädiktoren (kleine Subgruppen), dagegen bleibt nach der Kampagne nur das Präventionsverhalten in der Strandsituation als weiterer Prädiktor.

\footnotetext{
Literatur

Lausen B, Sauerbrei W, Schumacher M. Classification and regression trees (CART) used for the exploration of prognostic factors measured on different scales. In: Dirschedl P, Ostermann R, eds. Computational Statistics, Physica-Verlag, Heidelberg: 1994; 483-96.

2. Lausen B, Schumacher M. Maximally selected rank statistics. Biometrics 1992; 48: 73-85.

Koelmel K F, Pfahlberg A, Gefeller O. Melanomprävention durch Sonnenschutzmaßnahmen im Kindesalter. Hautarzt 1997; 48: 391-6.
} 